\title{
BMJ Open The impact of mass media interventions on tuberculosis awareness, health-seeking behaviour and health service utilisation: a systematic review protocol
}

\author{
Mweete D Nglazi, ${ }^{1,2}$ Linda-Gail Bekker, ${ }^{1}$ Robin Wood, ${ }^{1}$ Muki S Shey, ${ }^{3}$ \\ Olalekan A Uthman, ${ }^{4,5,6}$ Charles S Wiysonge ${ }^{6,7}$
}

To cite: Nglazi MD, Bekker L$\mathrm{G}$, Wood R, et al. The impact of mass media interventions on tuberculosis awareness, health-seeking behaviour and health service utilisation: a systematic review protocol. BMJ Open 2014;4:e004302. doi:10.1136/bmjopen-2013004302

- Prepublication history for this paper is available online. To view these files please visit the journal online (http://dx.doi.org/10.1136/ bmjopen-2013-004302)

Received 22 October 2013 Revised 4 December 2013 Accepted 6 December 2013

\section{CrossMark}

For numbered affiliations see end of article.

Correspondence to Mweete D Nglazi; Mweete. Nglazi@hiv-research.org.za

\section{ABSTRACT}

Introduction: Tuberculosis (TB) is a serious public health problem in many parts of the world. Strategies to curb the spread of TB must match the multifaceted nature of the epidemic. The use of mass media is one of the important strategies in communicating behavioural change in relation to TB prevention and the treatment. However, the benefits of this intervention are unclear. We, therefore, plan to conduct a systematic review on the effects of mass media interventions on TB awareness, health-seeking behaviour and health service utilisation.

Methods and analysis: We will preferably include randomised controlled trials (RCTs) in this systematic review. However, non-randomised studies will be included if there is an inadequate number of RCTs. We will perform electronic searches in PubMed, Scopus and other databases, along with manual searches. Articles written (or translated) in English and French and published between 1 January 1980 and 31 October 2013 will be eligible for inclusion in this review. The primary outcomes will be TB knowledge, attitudes and awareness, healthcare-seeking behaviour and service utilisation. The secondary outcomes will include stigma and discrimination against people with TB and the costs of the interventions. We will investigate clinical and statistical heterogeneity and pool studies judged to be clinically and statistically homogeneous. Relative risks will be calculated for dichotomous outcomes and mean differences for continuous outcomes, both with their corresponding $95 \% \mathrm{Cls}$.

Ethics and dissemination: The systematic review will use data that is not linked to individuals. The review findings may have implications for clinical practice and future research, and will be disseminated electronically and in print through peer-reviewed publications.

Protocol registration number: PROSPERO CRD42013005867

\section{INTRODUCTION}

Although there have been advances in the screening, diagnosis and treatment methods

\section{Strengths and limitations of this study}

- To our knowledge, this is the first systematic review protocol that will attempt to assess the impact of mass media interventions on tuberculosis awareness, health-seeking behaviour and health service utilisation.

- This study will help guide clinical practice and inform future studies on the most effective media platforms to use.

- Non-randomised studies with a high risk of bias and of low-quality evidence may be this study's limitation. We will, however, investigate the robustness of the results to risk of bias by excluding studies with a high risk of bias.

for the tuberculosis (TB) disease since Mycobacterium tuberculosis was first discovered, TB remains a serious public health problem in many parts of the world. Globally, there were an estimated 8.7 million new TB cases, and 1.4 million deaths from TB in 2011. ${ }^{1}$ The largest number of new cases of $\mathrm{TB}$ occurred in 22 low-income and middle-income countries, accounting for $80 \%$ of new cases globally in $2011{ }^{1}$

Directly observed treatment short course (DOTS) is the WHO recommended strategy for treating TB. However, the treatment alone is not sufficient to curb the spread of TB. Evidence suggests that cultural, environmental and politicoeconomic factors are important determinants of access to and use of TB services ${ }^{2}$, and so addressing the barriers to patients' access to and use of services can potentially turn the tide of the global TB epidemic. Therefore, strategies that promote early case detection and treatment adherence; raising awareness about $\mathrm{TB}$, reducing stigma, discrimination and delayed TB diagnosis or treatment as well as empowering people affected by TB and mobilising political commitment and resources for $\mathrm{TB}$ are 
important to curb the spread of $\mathrm{TB}^{3}{ }^{3}$ The advocacy, communication and social mobilisation (ACSM) activities recommended by the WHO is one such strategy. ${ }^{3}$ However, ASCM activities are most effective when delivered in combination and not separately. ${ }^{3}$

Mass media campaigns are widely used to disseminate information to vast audiences through the use of media, such as television, radio, newspapers, Internet, books, posters and billboards. They can play an important role in ACSM activities. Media advocacy involves using mass media in communicating TB-related health information to the public so as to raise awareness on TB-related issues and problems. ${ }^{3}$ The mass media campaigns may also be used to convey behaviour-change messages that aim to change the publics' knowledge, attitudes and practices. ${ }^{3}$

Mass media interventions have proven effectiveness in changing individuals' behaviour ${ }^{4-8}$ and healthcare utilisation $^{9}$, reducing stigma ${ }^{10}$ and raising awareness of the signs and symptoms of other diseases. ${ }^{11}$ With respect to $\mathrm{TB}$, mass media interventions have the potential to assist in generating knowledge about $\mathrm{TB}$, promoting awareness on the services that exist for TB diagnosis, prevention and treatment to assist with early diagnosis, treatment compliance and stigma reduction.

However, while systematic reviews on the impact of mass media interventions for promoting HIV testing, ${ }^{4}{ }^{5}$ smoking prevention, ${ }^{6}$ healthcare utilisation ${ }^{9}$ and reducing mental health-related stigma ${ }^{10}$ have already been undertaken, there are no systematic reviews of the impact of mass media interventions in TB management. We, therefore, plan to conduct a systematic review on the impact of mass media interventions on TB awareness, health-seeking behaviour and health service utilisation. A secondary aim is to examine the impact of mass media interventions on stigma and discrimination.

\section{METHODS AND ANALYSIS}

\section{Criteria for considering studies for review \\ Types of studies}

We will include randomised controlled trials (RCTs). However, non-randomised studies will be considered if there are inadequate number of RCTs (ie, less than five). We will include the following types of nonrandomised studies:

Non-RCTS;

Controlled before and after studies (CBA);

Interrupted time series studies (ITS), that is, studies in which there are at least three points of data collection before and after the intervention and the intervention occurs at a clearly defined time point;

Prospective cohort studies;

Retrospective cohort studies if baseline exposure data were collected at the time of baseline of the study.

The aforementioned studies may be useful in evaluating the effects of the mass media interventions when it is difficult to randomise.
Uncontrolled before and after studies will not be included in the review because secular trends or sudden attributes will make it difficult to attribute the observed changes to the mass media intervention. ${ }^{12}$ We will also exclude uncontrolled studies with postintervention measurements but no baseline measurements.

\section{Types of participants}

Participants will be the general public, specific target groups ( people living with HIV, healthcare workers, mine workers, prisoners, pregnant women, multidrug-resistant (MDR) TB suspects, household contacts of confirmed patients with $\mathrm{TB}$ and people with risk factors such as diabetes, smoking, alcohol or drug use) and patients with TB. These participants will be from all age groups (children and adults).

\section{Types of interventions}

Mass media interventions use a range of methods to communicate a message to the general public or specific groups. The media include radio, television, internet, mobile phone, print (newspapers, magazines, booklets, leaflets, posters and pamphlets), films, documentaries, billboards, folk media-like street dramas or a combination of these. For the proposed systematic review, we will include studies that report on any mass media intervention aimed to either raise TB awareness and knowledge levels or raise health service utilisation rates or other health-seeking behaviour or reduce stigma and discrimination levels and delivered in formats seeking to meet the needs of the general and specific target audiences (see participants above).

We will exclude interventions based on scientific media such as professional journals, leaflets and booklets specifically targeted to healthcare workers. We will also exclude interventions based on the use of media such as video, booklets and leaflets in a healthcare setting for individual patient information purposes. We will exclude studies of mass media interventions that have only been reported in terms of secondary outcomes. Lastly, we will exclude mass media interventions coupled with other interventions because it will be difficult to separate out the effects of the multiple interventions occurring simultaneously.

The comparison will be no intervention, preintervention waiting list control or an alternative intervention.

\section{Types of outcome measures}

\section{Primary outcome}

The primary outcomes in this review are:

Changes in knowledge, attitudes, awareness and opinions about TB and health-seeking behaviour intentions for TB.

Changes in TB service utilisation (TB testing uptake; TB case detection rate; the number of new cases of TB; the number of direct smears processed by laboratories; TB treatment adherence rates; retention rates 
and rates of surgical procedures used in $\mathrm{TB}$ the diagnosis or treatment, etc).

\section{Secondary outcomes}

The secondary outcomes include:

Changes in the level of stigma (aspects such as negative attitudes towards the perceived behaviour of people with TB and lack of knowledge about TB) and discrimination against people with TB.

Cost of the intervention.

Objectively measured outcomes data as reported in included studies will be considered acceptable.

\section{Definitions}

In the context of this systematic review, TB service utilisation is defined broadly to include the use of preventative or curative TB treatment, TB diagnostic tests and surgical procedures used in $\mathrm{TB}$ diagnosis or treatment.

\section{Search methods for identification of studies \\ Electronic searches}

MDN, with the help of a university librarian, will perform electronic searches in PubMed, Scopus, Cochrane Central Register of Controlled Trials (CENTRAL), ISI Web of Science (Science Citation Index), Africa-Wide Information, Cumulative Index of Nursing and Allied Health (CINAHL) and PsycINFO. Table 1 presents the PubMed search strategy. Slight modifications of this strategy will be used for the other databases. Articles written in or translated into English and French and published from January 1980 (the advent of the new wave of TB infections related to HIV/AIDS and the subsequent emergence of drug-resistant TB from the misuse or mismanagement of anti-TB treatment) to 31 October 2013 will be eligible for inclusion in this review.

\section{Searching other sources}

Manual searches will include scanning the reference lists of relevant studies, specialist journals and conference proceedings. Information on unpublished or ongoing studies will be sought through the WHO International Clinical Trial Registry Platform, Clinicaltrials.gov, Pan African Clinical Trials Registry (PACTR) and through correspondence with experts within the TB field. The websites tb.org and http://www.stoptb.org will be sought for available material.

\section{Data collection and analysis}

The methodology for data collection and analysis will be based on the guidance of the Cochrane Handbook of Systematic Reviews for Interventions. ${ }^{13}$

\section{Selection of studies}

A screening form will be created for this review. This screening form will be piloted to ensure that the inclusion criteria are adhered to and consistently applied by all review authors. Two review (MDN and CSW) authors will independently scan search outputs and retrieve potentially eligible studies using the piloted screening form. One review author will obtain the full text articles deemed to be relevant, and MDN and CSW will independently assess these for eligibility using the aforementioned criteria. Then, the studies excluded will be listed with the reason for exclusion. If disagreements occur between assessors, they will be resolved through discussion and consensus. If the disagreement persists, a third author (LGB, RW, MS, OU) will be consulted.

\section{Data extraction and management}

A standardised data extraction form will be developed for this review. We will pilot the form on three studies to assess its completeness and usability. Two review authors (MDN and CSW) will independently extract data using the standardised data extraction form, resolving discrepancies by discussion and consensus. When there is a difference of opinion, a third review author will be consulted. MDN will enter the final data into Review Manager V.5.2 statistical software (The Cochrane Collaboration, Copenhagen, Denmark). CSW will crosscheck the data entered to ensure that there are no data entry errors.

The following data will be extracted from the included studies for use in assessing risk of bias.

Characteristics of the study: study setting and location; and the number of study sites (when applicable), study design, the method of recruitment of participants and the funding source.

Patient characteristics: age, sex and ethnicity of the participants; number of participants.

Characteristics of the intervention: type and duration of the intervention.

Independent variables that are studied.

Outcome data in the intervention and control group.

\section{Assessment of risk of bias in included studies}

Two review authors will independently assess risk of bias in each included study. We will use the Cochrane Risk of Bias tool in the assessment. ${ }^{13}$ Separate criteria will be used to assess RCTs and non-randomised studies. For RCTs, we will assess risk of bias using the following key criteria: random sequence generation; allocation concealment; blinding of participants and personnel; blinding of outcome assessors; incomplete outcome data; selective outcome reporting; other sources of bias and overall risk of bias. ${ }^{13}$ For non-randomised studies, we will focus on specific outcomes of the study (eg, outcomes assessments) and the extent to which the studies are susceptible to bias (eg, selection, performance, detection, attrition and reporting biases) in assessing risk of bias. ${ }^{13}$ In addition to using the Cochrane risk of bias tool, we will also use the Newcastle-Ottawa Scale (NOS) to assess risk of bias in non-randomised studies. ${ }^{14}$ The NOS provides criteria for making judgments about risk of bias in the following areas: selection of study groups, comparability of groups and ascertainment of outcomes (in the case of cohort studies). ${ }^{14}$ Therefore, this instrument 


\section{Table 1 PubMed search strategy, modified as appropriate for use in other database}

\begin{tabular}{|c|c|}
\hline No & Query \\
\hline$\# 1$ & tuberculosis $[\mathrm{MeSH}]$ \\
\hline \#2 & Tuberculosis [tiab] \\
\hline \#3 & \#1 OR \#2 \\
\hline \#4 & "mass media" [MeSH] OR "mass media" [tiab] \\
\hline \#5 & "health communication" [MeSH] OR "health communication*" [tiab] \\
\hline \#6 & communication [MeSH] OR communication* [tiab] \\
\hline \#7 & "multimedia" [tiab] \\
\hline \#8 & multimedia [tiab] \\
\hline \#9 & "mass communication" [tiab] \\
\hline \#10 & "audiovisual equipment" [tiab] \\
\hline \#11 & "patient information" [tiab] \\
\hline \#12 & "visual information" [tiab] \\
\hline \#13 & radio [tiab] \\
\hline \#14 & television [tiab] \\
\hline \#15 & leaflet $^{*}[$ tiab] \\
\hline \#16 & poster* $^{*}$ [tiab] \\
\hline \#17 & pamphlet $^{\star}[$ tiab] \\
\hline \#18 & "print media" [tiab] \\
\hline \#19 & "printed media" [tiab] \\
\hline \#20 & skit $^{*}[$ tiab] \\
\hline \#21 & "talk show" [tiab] \\
\hline \#22 & broadcast $^{\star}[$ tiab] \\
\hline \#23 & film* [tiab] \\
\hline \#24 & telecommunication* [tiab] \\
\hline \#25 & internet [MeSH] OR internet [tiab] \\
\hline \#26 & Patient advocacy [MeSH] OR advocacy [tiab] OR "social mobilization" [tiab] OR "ACSM" [tiab] \\
\hline \#27 & $\begin{array}{l}\# 4 \text { OR \#5 OR \#6 OR \#7 OR \#8 OR \#9 OR \#10 OR \#11 OR \#12 OR \#13 OR \#14 OR \#15 } \\
\text { OR \#16 OR \#17 OR \#18 OR \#19 OR \#20 OR \#21 OR \#22 OR \#23 OR \#24 OR \#25 OR \#26 }\end{array}$ \\
\hline \#28 & \#27 AND \#3 \\
\hline \#29 & "health behavior" [MeSH] OR "health behavior" [tiab] \\
\hline \#30 & "health promotion" [MeSH] OR "health promotion" [tiab] \\
\hline \#31 & "health education" [MeSH]] OR "health education" [tiab] \\
\hline \#32 & "patient education as topic" [MeSH] \\
\hline \#33 & $\begin{array}{l}\text { "health knowledge, attitudes, practice" [MeSH] OR knowledge [tiab] OR attitude* [tiab] OR } \\
\text { practice* [tiab] }\end{array}$ \\
\hline \#34 & "awareness" [MeSH] OR "awareness [tiab] \\
\hline \#35 & "Health Services/utilization" [MeSH] \\
\hline \#36 & "Health Services Research" [MeSH] \\
\hline \#37 & "Patient Acceptance of Health Care" [MeSH] OR "health seeking behavior" [tiab] \\
\hline \#38 & \#29 OR \#30 OR \#31 OR \#32 OR \#33 OR \#34 OR 35 OR \#36 OR \#37 \\
\hline \#39 & \#28 AND \#38 \\
\hline \#40 & Humans $[\mathrm{MeSH}]$ \\
\hline \#41 & \#40 AND \#39 \\
\hline
\end{tabular}

helps to assess the quality of non-randomised studies so that they can be included in a systematic review.

The risk of bias table available in Review Manager V.5.2 software (The Cochrane Collaboration, Copenhagen, Denmark) will need to be operationalised in order to be used for RCTs and non-randomised studies (in the case of non-RCTs, CBAs and cohort studies) according to the aforementioned criteria. ${ }^{13} 14$ Certain items in the Risk of Bias table, for example, random sequence generation will not be applicable for non-randomised studies. Other biases will include four areas: comparability of cohorts for baseline characteristics and outcome measures on the basis of design and analysis; selection of the non-exposed cohort; protection against contamination and ascertainment of exposure.

For assessing the methodological quality of ITS studies, we will use the criteria developed by the Cochrane Effective Practice and Organization of Care (EPOC) Review Group to assess the risk of bias. ${ }^{15}$ These criteria are as follows:

Was the intervention independent of other changes? Was the shape of the intervention effect prespecified? Was the intervention unlikely to affect data collection? Was knowledge of the allocated interventions adequately prevented during the study?

Were incomplete outcome data adequately addressed? 
Was the study free from selective outcome reporting?

Was the study free from other risks of bias?

Studies will be scored as low, high or unclear risk of bias. The two authors will resolve disagreements in the assessment of risk of bias by discussion and consensus, consulting a third author to resolve any persistent disagreements.

\section{Measures of treatment effect}

The outcomes of interest will be either dichotomous or continuous. Relative risks will be calculated for dichotomous outcomes and mean differences for continuous outcomes, both with their corresponding $95 \%$ CIs.

\section{Assessment of heterogeneity}

Clinical heterogeneity will be investigated by examining the types of participants, interventions and outcomes in each study. Then, we will pool studies judged to be clinically homogenous. Statistical heterogeneity will be investigated using the $\chi^{2}$ test of homogeneity and quantified using the $\mathrm{I}^{2}$ statistic. ${ }^{16}{ }^{17}$ If the study results are found to be statistically homogeneous (ie, heterogeneity $\mathrm{p}>0.1$ ), we will pool them using the fixed-effect meta-analysis. Otherwise, we will use random-effects meta-analysis. The grading of recommendations, assessment, development and education (GRADE) approach will be used to assess the quality of the body of evidence. ${ }^{18} 19$ This method results in the assessment of the quality of the body of evidence as high, moderate, low or very low. The evidence is considered of high quality if further research is very unlikely to change our confidence in the estimate of the effect; and moderate quality if further research is likely to have an important impact on our confidence in the estimate of the effect and may change the estimate. The low-quality evidence implies that further research is likely to have an important impact on our confidence in the estimate of the effect and is likely to change the estimate, and very low quality implies that we have very little confidence in the effect estimate.

\section{Dealing with missing data}

Relevant missing data will be sought by contacting the corresponding authors of included studies. We will use the last observation carried forward to handle missing data that cannot be obtained. ${ }^{13}$

\section{Data analysis}

Data analysis will be conducted using Review Manager V.5.2 statistical software (The Cochrane Collaboration, Copenhagen, Denmark). Where studies are found to be clinically and statistically homogeneous, we will pool the studies in a meta-analysis. For dichotomous outcomes, we will combine the results and calculate the risk ratio and $95 \%$ CIs. For continuous outcomes that are measured on the same scale, we will combine the mean differences to calculate the mean difference and SD. For continuous outcomes that are not measured on the same scale, we will standardise the measurements on a uniform scale before they can be pooled in a meta-analysis. Then we will calculate the standardised mean difference and $95 \%$ CIs.

\section{Subgroup analysis}

We shall explore stratification by the following subgroups:

Age (children, adolescents, adult or mixed populations);

Type of mass media intervention;

Study setting (low-income and middle-income countries vs high-income countries).

These subgroups were chosen because we anticipate statistical heterogeneity due to anticipated differences between study populations and interventions. For example, we anticipate that age will have an impact on the interpretation of TB-related messages communicated through mass media. Children and adults are more likely to positively accept TB messages communicated in an innovative, fun and engaging manner that promote internalised decision-making and ultimately influence their TB-related behaviour, compared with adults.

\section{Sensitivity analysis}

We will conduct sensitivity analyses to assess the effect of missing data on our primary meta-analyses. When we find a study with missing data, we will first perform available cases analysis, followed by sensitivity analyses according to imputations (ie, from assuming that all missing data are failures to assuming that all missing data are successes). We will also conduct sensitivity analyses to investigate the robustness of the results to risk of bias (ie, excluding trials with high risk of bias) and method of meta-analysis (ie, random-effects vs fixed-effect). For sensitivity analyses, we will define 'high risk of bias' based on each of three criteria, namely inadequate concealment of group allocation, inadequate blinding of outcome assessment and differential loss to follow-up (ie, differences in the proportion of incomplete outcome data across intervention groups, where availability of outcome data is determined by the participants' true outcomes).

\section{Ethics and dissemination}

The systematic review did not require ethical approval because the data cannot be linked to an individual. The findings of this systematic review will have important implications for clinical practice and research. The review will shed light on the potential use of mass media interventions to promote knowledge, attitudes and opinions about TB and encourage the use of TB services by those engaged in promoting better uptake of health information in clinical practice. Future studies may learn how to create effective media messages about TB, what is the most effective media platform to use and whether media messages have different impacts on different target audiences. The findings of this systematic review will be disseminated electronically and in print by peerreviewed publication. 
Author affiliations

${ }^{1}$ Institute of Infectious Disease and Molecular Medicine and the Department of Medicine, The Desmond Tutu HIV Centre, Faculty of Health Sciences, University of Cape Town, Cape Town, South Africa

${ }^{2}$ International Union against Tuberculosis and Lung Disease, Paris, France ${ }^{3}$ South African Endpoint Laboratory, Aeras Global TB Vaccine Foundation, Cape Town, South Africa

${ }^{4}$ Division of Health Sciences, Warwick-Centre for Applied Health Research and Delivery (WCAHRD), Warwick Medical School, The University of Warwick, Coventry, UK

${ }^{5}$ International Health Group, Liverpool School of Tropical Medicine, Liverpool, Merseyside, UK

${ }^{6}$ Centre for Evidence-based Health Care, Department of Interdisciplinary Health Sciences, Faculty of Medicine and Health Sciences, Stellenbosch University, Cape Town, South Africa

${ }^{7}$ Division of Community Health, Department of Interdisciplinary Health Sciences, Faculty of Medicine and Health Sciences, Stellenbosch University, Cape Town, South Africa

Contributors MDN and CSW contributed to the conception and design of the study protocol. All the authors were involved in the drafting of this study protocol and have given their approval for publication.

Competing interests None.

Provenance and peer review Not commissioned; externally peer reviewed.

Open Access This is an Open Access article distributed in accordance with the Creative Commons Attribution Non Commercial (CC BY-NC 3.0) license, which permits others to distribute, remix, adapt, build upon this work noncommercially, and license their derivative works on different terms, provided the original work is properly cited and the use is non-commercial. See: http:// creativecommons.org/licenses/by-nc/3.0/

\section{REFERENCES}

1. World Health Organization (WHO). Global Tuberculosis Report. Geneva: World Health Organization, 2012. http://www.who.int/tb/ publications/global_report/gtbr12_main.pdf (accessed 2 Sep 2013).

2. Ho MJ. Sociocultural aspects of tuberculosis: a literature review and a case study of immigrant tuberculosis. Soc Sci Med 2004;59:753-62.

3. World Health Organization (WHO). Advocacy, communication and social mobilization (ACSM) for tuberculosis control: a handbook for country programmes. Geneva: World Health Organization, 2007. http://www stoptb.org/assets/documents/resources/publications/ acsm/ACSM_Handbook.pdf (accessed 2 Oct 2013).

4. Vidanapathirana J, Abramson MJ, Forbes A, et al. Mass media interventions for promoting HIV testing. Cochrane Database Syst Rev 2005;(3):CD004775.

5. Bala MM, Strzeszynski L, Topor-Madry R, et al. Mass media interventions for smoking cessation in adults. Cochrane Database Syst Rev 2013;(6):CD004704.

6. Brinn MP, Carson KV, Esterman AJ, et al. Mass media interventions for preventing smoking in young people. Cochrane Database Syst Rev 2010;(11):CD001006.

7. Durkin S, Brennan E, Wakefield M. Mass media campaigns to promote smoking cessation among adults: an integrative review. Tob Control 2012;21:127-38.

8. Elder RW, Shults RA, Sleet DA, et al. Effectiveness of mass media campaigns for reducing drinking and driving and alcohol-involved crashes: a systematic review. Am J Prev Med 2004;27:57-65.

9. Grilli R, Ramsay C, Minozzi S. Mass media interventions: effects on health services utilisation. Cochrane Database Syst Rev 2002;(1): CD000389.

10. Clement S, Lassman F, Barley E, et al. Mass media interventions for reducing mental health-related stigma. Cochrane Database Syst Rev 2013;(7):CD009453

11. Lecouturier J, Rodgers $\mathrm{H}$, Murtagh MJ, et al. Systematic review of mass media interventions designed to improve public recognition of stroke symptoms, emergency response and early treatment. BMC Public Health 2010;10:784.

12. Grimshaw J, Campbell M, Eccles M, et al. Experimental and quasi-experimental designs for evaluating guideline implementation strategies. Fam Pract 2000;17(Suppl 1):S11-16

13. Higgins JPT, Green S, eds. Cochrane handbook for systematic reviews of interventions Version 5.1.0 [updated March 2011]. The Cochrane Collaboration, 2011. http://www.cochrane-handbook.org

14. Stang A. Critical evaluation of the Newcastle-Ottawa scale for the assessment of the quality of nonrandomized studies in meta-analyses. Eur J Epidemiol 2010;25:603-5.

15. Cochrane Effective Practice and Organization of Care Group (EPOC). Data Collection Checklist, 2008. http://epoc.cochrane.org/ sites/epoc.cochrane.org/files/uploads/datacollectionchecklist.pdf (accessed 28 Nov 2013)

16. Higgins JP, Thompson SG. Quantifying heterogeneity in a meta-analysis. Stat Med 2002;21:1539-58.

17. Higgins JP, Thompson SG, Deeks JJ, et al. Measuring inconsistency in meta-analyses. BMJ 2003;327:557-60.

18. Balshem $\mathrm{H}$, Helfand $\mathrm{M}$, Schunemann $\mathrm{HJ}$, et al. GRADE guidelines: 3. Rating the quality of evidence. J Clin Epidemiol 2011;64:401-6.

19. Guyatt GH, Oxman AD, Vist GE, et al. GRADE: an emerging consensus on rating quality of evidence and strength of recommendations. BMJ 2008:336:924-6. 